\title{
Segregation of Quarks within the Nucleon
}

\author{
Zbigniew Dziembowski \\ Institute for Nuclear Theory, University of Washington \\ HN-12, Seattle, Washington 98195, USA
}

\section{PREPARED FOR THE U.S. DEPARTMENT OF ENERCY UNDER GRANT DE-FG06-90ER40561}

This report was prepared as an account of work sponsored by the United States Government. Neither the United States nor any agency thereof, nor any of their employees, makes any warranty, express or implied, or assumes any legal liability or responsibility for the accuracy, completeness, or usefulness of any information, apparatus, product, or process disclosed, or represents that its use would not infringe privately owned rights. Reference herein to any specific commercial product, process, or service by trade name, mark, manufacturer, or otherwise, does not necessarily cunstitute or imply its endorsement, recommendation, or favoring by the United States Government or any agency thereof. The views and opinions of authors expressed herein do not necessarily state or reflect those of the United States Government or any agency thereof.

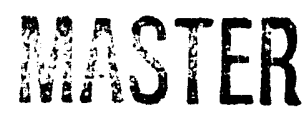




\title{
Segregation of Quarks within the Nucleon
}

\author{
Zbigniew Dziembowski ${ }^{\dagger}$ \\ Institute for Nuclear Theory, HN-12 \\ University of Washington, WA 98195
}

\begin{abstract}
Measurements of the nucleon form factor and structure function seem to indicate an inhomogeneous distribution of flavor, charge and spin within the nucleon. It is argued that the ordinary three-quark model with a spin-spin force of the type suggested by QCD can explain the inhomogeneity as seen at different resolutions. This agreement suggests a specific bound quark picture of the nucleon structure with a positive core of $u$ and $d$ quarks in a spin-0 state of ms radius $0.17 \pm 0.01 \mathrm{fm}^{2}$ and an outer layer of a linear size $\sim 1 \mathrm{fm}$ where the polarized $u$ (in the proton) or $d$ (in the neutron) is orbiting.
\end{abstract}

\footnotetext{
'Permanent address: Department of Physics, Temple University, Philadelphia, PA
} 19122 
It was a set of experiments at SLAC in the late 1960's on deep inelastic scattering off proton and deuterium targets that showed the nuclion to be made of point-like objects. Further studies with neutrinos, polarized electrons and muons established the spin, charge and baryon number of the point-like objects and showed they were quarks. It was also recognized that these experiments not only detect quarks but directly measure their distribution within the target. In more technical terms, they measure different light-cone quark correlation functions in the target ground state. One aspect which has, however, eluded description for many years is the connection between these momentum distributions and the spatially inhomogeneous distribution of flavor and spin within the nucleon that emerges from quark potential models.

In this Letter I re-examine the low-energy nucleon structure which follows from the nonrelativistic constituent quark model. I choose the successful QCD-motivated form of the quark model developed by Isgur and Karl [1], but the conclusions reached here are not restricted to this particular representation. There are good reasons to believe that any quark model that consists $c \dot{f}$ a combination of two dynamical elements: a long-distance confining potential and a short-distance spin-spin force of the type suggested by QCD should predict a spatially innomogeneous distribution of flavor and spin within the nucleon. Once the emerging quark-diquark picture agrees with such low scale features of the electromagnetic nucleon structure as charge radii, it also gives, after being transformed to the light-cone, the flavor-spin asymmetric quark momentum distributions needed to explain the difference in neutron and proton structure functions or the proton polarization asymmetry measured in the DIS experiments.

I start with a review of the essential elements of the quark potential model 
of Ref. [1] and then show how the model's dynamics leads in general to a spatial segregation of quarks within the nucleon.

For a low-momentum-scale description of the nucleon ground state one needs three quarks of two flavors ( $u u d$ in the proton and $d d u$ in the neutron) with effective masses $m \simeq m_{N} / 3$. Quarks are spin-1/2 particles and carry a color quantum number that influences the permutation symmetry of the nucleon wave function.

The model finds two dynamical elements being dominant for the nucleon ground state properties. First, there is a long-distance, spin-independent confining potential that determines the overall size of the system and gives a symmetric momentum spread to the three quark state of the order of 250 $\mathrm{MeV}$ [2]. In addition, there is the contact hyperfine spin-spin interaction motivated by QCD one-gluon exchange and responsible for breaking the nucleon-delta mass degeneracy.

This force is repulsive for quark pairs in a spin-1 state and attractive for those in a spin-0 state. It is known to make the $\Delta(1232)$ (with spin $3 / 2$ ) more massive than the nucleon (with spin 1/2). But what is more important here is that the force also modifies the nucleon wave function.

Refs. [3, 4] contain perturbation theory estimates showing how the hyperfine interaction modifies the totally symmetric wave function of the harmonic-oscillator confining potential. Using a spin-flavor $\mathrm{SU}(6)$ basis, one finds that the dominant effect of the interaction is the mixing of the $N^{2} S_{M}\left(\left[70,0^{+}\right]\right)$configuration into the pure $N^{2} S_{S}\left(\left[56,0^{+}\right]\right)$nucleon. Thus the physical ground state is of the form

$$
\left.|N>\simeq \cos \varphi| N_{S}\right\rangle+\sin \varphi \mid N_{M}>\text {, }
$$

for which the perturbation theory calculation of Refs. [4] yields $\varphi \simeq-15^{\circ}$. 
Now to reveal two-quark correlations in the nucleon wave function, I first abandon $\mathrm{SU}(6)$ with its merely historical significance and re-write the mixture in the " $u d s$ " basis [5]. Thus the relevant components are

$$
\left|N_{S}\right\rangle=\chi^{\lambda} \psi^{S}, \quad\left|N_{M}\right\rangle=\frac{1}{\sqrt{2}}\left(\chi^{\rho} \psi^{\rho}-\chi^{\lambda} \psi^{\lambda}\right),
$$

where the $\chi$ 's are the usual spin- $\frac{1}{2}$ wave functions $\chi^{\rho}=\left(s_{2}-s_{1}\right) / \sqrt{2}$, $\chi^{\lambda}=\left(2 s_{3}-s_{1}-s_{2}\right) / \sqrt{6}$, with $s_{1}=\downarrow \uparrow \uparrow, s_{2}=\uparrow \downarrow \uparrow, s_{3}=\uparrow \uparrow \downarrow$, and the $\psi$ 's are the harmonic-oscillator wave functions $\psi^{S}=A \exp \left(-\alpha^{2}\left\{\rho^{2}+\lambda^{2}\right\} / 2\right)$, $\psi^{\rho}=2 \alpha^{2} \rho \cdot \lambda \psi^{s} / \sqrt{3}, \psi^{\lambda}=\alpha^{2}\left(\rho^{2}-\lambda^{2}\right) \psi^{s} / \sqrt{3}$ with $\rho=\left(\mathbf{r}_{1}-\mathbf{r}_{2}\right) / \sqrt{2}$ and $\lambda$ $=\left(r_{1}+r_{2}-2 r_{3}\right) / \sqrt{6}$. Next, I abandon the symmetric harmonic-oscillator basis for the distorted one used by Isgur and Karl in their description of the baryon structure in the strange sector. The distorted harmonic-oscillator(DHO) basis is much better suited for describing the quark-diquark picture of the nucleon structure induced by the hyperfine interaction. The use of the DHO basis trades off the mixing angle of the perturbative calculation for a more intuitive deformation parameter defined below. The latter is unambiguously constrained by the experimental value of the neutron to proton charge radius ratio.

To visualize how the wave function is deformed, let me consider the nucleon as being initially in the symmetric Gaussian configuration $\mid N_{S}>$ produced by the harmonic oscillator part of the confining potential alone. In the spin state $\chi^{\lambda}=\left(s_{3}-s_{1}+s_{3}-s_{2}\right) / \sqrt{6}$ the first two quarks with the same flavor are in a spin-1 state whereas each $u-d$ fair is in a spin-0 state. This implies that the $u$ and $d$ quarks are attracted to each other by the spin interaction, but the odd one (i.e., $u$ quark in the proton or $d$ quark in the neutron) is driven further from the center of mass of the system. In the DHO basis, this effect can be parameterized through the use of a scale parameter 
$\alpha_{\rho}^{2}=\alpha^{2}(1+d)$ (corresponding to a smaller size) for the spin-0 pairs of $u-d$ quarks that is larger than the one $\alpha_{\lambda}^{2}=\alpha^{2}(1-d)$ for the coupling of the odd quark to this pair. After symmetrization with respect of the quantum numbers of the first two quarks, one gets

$$
|N\rangle=\left(s_{3}-s_{1}\right) \Phi(13,2)+\left(s_{3}-s_{2}\right) \Phi(23,1)
$$

where the asymmetric spatial wave function for the quark configuration $(12,3)$ is

$$
\Phi(12,3)=A \exp \left(-\frac{1}{2}\left\{\alpha_{\rho}^{2} \rho^{2}+\alpha_{\lambda}^{2} \lambda^{2}\right\}\right) .
$$

To buiid credibility for this simple model, I show its relation to the configuration mixing of Eq. (1). Expanding the wave function of Eq. (3) up to linear terms in the deformation parameter $d(>0)$, one gets

$$
|N\rangle=\chi^{\lambda} \psi^{S}-\frac{\sqrt{3} d}{2 \sqrt{2}} \frac{1}{\sqrt{2}}\left(\chi^{\rho} \psi^{\rho}-\chi^{\lambda} \psi^{\lambda}\right) \text {. }
$$

One can easily recognize in the above expression the mixture of $\left[56,0^{+}\right]$and $\left[70,0^{+}\right]$( written in the "uds" basis) with

$$
\tan \varphi=-\frac{\sqrt{3}}{2 \sqrt{2}} d
$$

To estimate the deformation parameter $d$ and the Gaussian scale $\alpha$ in (4) I calculate the nucleons' $m s$ charge radii. With simple quark additivity one gets

$$
\left\langle r^{2}\right\rangle_{p}^{c h}=\frac{1}{\alpha^{2}}\left(1+\frac{1}{2} d\right) f(d), \quad\left\langle r^{2}\right\rangle_{n}^{c h}=\frac{1}{\alpha^{2}}\left(-\frac{1}{2} d\right) f(d) .
$$

where $f(d)=\left[2\left(1-d^{2}\right)^{-5 / 2}+\left(1-d^{2} / 4\right)^{-5 / 2}\right] /\left[2\left(1-d^{2}\right)^{-3 / 2}+\left(1-d^{2} / 4\right)^{-3 / 2}\right]$. As expected, the spin-spin interaction that drives the odd quark further from the center of mass makes the proton charge radius bigger (compared to the symmetric limit of $\left.1 / \alpha^{2}\right)$ and yields the proper sign for $\left\langle r^{2}\right\rangle_{n}^{\text {ch }}$. Using 
input from the experimental data: $\left\langle r^{2}\right\rangle_{p}^{c h}=0.743 \pm 0.02 \mathrm{fm}^{2}$ (Ref. [6]) and $\left\langle r^{2}\right\rangle_{n}^{c h}=-0.117 \pm 0.002 \mathrm{fm}^{2}$ (Ref. [7]), one obtains $d=0.372 \pm 0.020$ and $\alpha=264 \pm 11 \mathrm{MeV}$. With these values the present model produces the correct values for the neutron and proton charge radii and yields a wave function which differs from that of the Isgur-Karl model only by terms of order $d^{2}$ provided one neglects (small) contributions from the $N^{2} S_{S^{\prime}}$ and $N^{4} D_{M}$ configurations.

Let me mention at this point that it has been recognized for many years that a spatially dependent spin-spin interaction that is more repulsive for quarks with parallel rather than antiparallel spins may induce a segregation of charge within the neutron [8]. This possibility has been used with success in a quark model with a hyperfine interaction to explain quantitativeiy the charge radius of this particle $[3,4]$. Note, however, that the above dynamical argument about segregation is far more general. In fact it implies that the short distance hyperfine interaction together with Fermi-Dirac statistics leads to a spatial segregation of both flavor and spin distributions within the proton and neutron. Thus, one should expect more spectacular manifestations than just the negative charge radius of the neutron.

To see the segregation of quarks in configuration space, I calculate the spin average $(u(r), d(r))$ and spin weighted $(\Delta u(r), \Delta d(r))$ probabilities of finding a $u$ or $d$ quark at a distance $r$ from the center of mass of the nucleon. Fig. 1 gives the neutron charge density $\rho_{n}(r)=\left(e_{u} d(r)+e_{d} u(r)\right)$ together with the integral $\int_{0}^{r} \rho_{n}(\mathbf{x}) \mathbf{x}^{2} d^{3} x$. The latter quantity exhibits the distance over which the total neutron ms charge radius is built up. Fig. 2 shows the flavor ssymmetry $F_{2}^{n}(r) / F_{2}^{p}(r)=\left(e_{u}^{2} d(r)+e_{d}^{2} u(r)\right) /\left(e_{u}^{2} u(r)+e_{d}^{2} d(r)\right)$ and the proton spin asymmetry $A_{1}^{p}(r)=\left(e_{u}^{2} \Delta u(r)+e_{d}^{2} \Delta d(r)\right) /\left(e_{u}^{2} u(r)+\right.$ $\left.e_{d}^{2} d(r)\right)$, respectively. These quantities are compared with the predictions of 
the symmetric model of $; N_{S}>$.

All distributions suggest the existence of a soft positive $u-d$ core in the spin-0 state and a broad layer of linear size approximately $\sim 1 \mathrm{fm}$ where the polarized $u$ (in the proton) or $d$ (in the neutron) is orbiting. One can now estimate the ms charge radius of the core as $\left.\left\langle r^{2}\right\rangle_{\text {core }}^{\text {ch }} \equiv<r_{3}^{2}\right\rangle / 3=$ $\left(<r^{2}>_{p}^{c h}+2<r^{2}>_{n}^{c h}\right) / 3$. Substituting the experimental values from Refs. [6, 7], one gets $\left\langle r^{2}\right\rangle_{\text {core }}^{c h}=0.17 \pm 0.01 \mathrm{fm}^{2}$. This result gives a useful hint as to why the quark-diquark approximation (as old as the quark model) seems to be so useful in some model calculations [9].

In the second part of this Letter I examine how the quark segregation caused by the spatially dependent spin-spin interaction shows up in the lightcone correlation functions. For this purpose I need to establish a connection between the low energy picture and the nucleon structure as revealed by DIS experiments. The link is provided by the theoretical framework of the operator product expansion that allows a separation of physical quantities into factors corresponding to the short- and long-distance physics. Perturbative QCD is the proper tool to apply to the former but a non-perturbative bound-state solution of QCD must be called upon to attempt any kind of description of the $x$-dependence of the structure functions [10].

But what :s the relevance of the quark model for the bound-state problem in QCD? In a quark model the nucleon is assumed to consist of three valence quarks, but in QCD the quarks have a structure due to their radiating gluons and quark-antiquark pairs. Thus, already at a scale of few $Q^{2}$, one needs many Fock-state components for any realistic description of the DIS data. It is possible, however, to reconcile these two pictures. The idea was first suggested in the early days of QCD [11] and is based on the observation that when the evolution equations for the moments of the parton distributions are 
run backwards, the $\simeq 16 \%$ of the momentum carried by $q \bar{q}$ sea and $\simeq 44 \%$ carried by the gluons in the nucleon seen at $Q^{2} \simeq O\left(16 \mathrm{GeV}^{2}\right)$ mostly disappear at some low scale $\simeq O\left(1 \mathrm{GeV}^{2}\right)$ [12]. Using the MIT bag-model version of the quark non-perturbative dynamics, Jaffe and Ross [13] have showed show that the three quark model can be used to calculate the leading twist non-singlet structure functions at low scale $O\left(1 \mathrm{GeV}^{2}\right)$. When these low-scale structure functions are later evolved to $\left.Q^{2}>Q_{1} \mathrm{GeV}^{2}\right)$, they successfully compare with experiment. Encouraged by this result, I shall use the same method to link the low-scale three-quark wave function of the QCD-inspired quark model to the non-singlet structure functions at high $Q^{2}$. However, to diminish the influence of the perturbative evolution on my conclusion, I choose to calculate here only ratios of different structure functions in the valence region of $x>0.2$. At the same time, taking ratios should also reduce the influence of the Gaussian ansatz of Eq. (4).

I take the static nucleon wave function of Eq.(3), transform it to the momentum space and then change to light-cone variables. This envolves a Melosh-type boost of the static spin wave function [14] $s_{2}-s_{1}$ that gives

$$
J(12,3)=\bar{u}_{\lambda_{1}}\left(m_{N}+p_{\mu} \gamma^{\mu}\right) \gamma_{5} v_{\lambda_{2}} \bar{u}_{\lambda_{3}} u_{N}
$$

Then, the use of the Brodsky-Huang-Lepage prescription for the Gaussian wave function leads to

$$
\Phi(12,3)=\exp \left(-\frac{1}{6 \alpha_{\rho}^{2}}\left[\frac{m_{1}^{2}+q_{1}^{2}}{x_{1}}+\frac{m_{2}^{2}+q_{1}^{2}}{x_{2}}\right]-\frac{1}{6 \alpha_{\lambda}^{2}}\left[\frac{m_{3}^{2}+Q_{1}^{2}}{x_{3}}+\frac{Q_{1}^{2}}{x_{1}+x_{2}}\right]\right)
$$

where $\mathrm{q}_{\perp}=\left(x_{2} \mathbf{k}_{\perp 1}-x_{1} \mathbf{k}_{\perp 2}\right) /\left(x_{1}+x_{2}\right)$ and $\mathbf{Q}_{\perp}=\left(x_{3}\left(\mathbf{k}_{\perp 1}+\mathrm{k}_{\perp 2}\right)-\left(x_{1}+\right.\right.$ $\left.\left.x_{2}\right) \mathrm{k}_{\perp 3}\right) /\left(x_{1}+x_{2}+x_{3}\right)$ are the light-cone relative transverse momenta. Details of the procedure are described elsewhere [15]. Finally one arrives at the 
following low-scale valence light-cone wave function

$$
\Psi\left(x_{i}, \mathbf{k}_{\alpha_{i}}, \lambda_{i}\right)=(J(13,2) \Phi(13,2)+J(23,1) \Phi(23,1)) / \sqrt{x_{1} x_{2} x_{3}},
$$

where $x_{i}=k_{i}^{+} / p_{N}^{+}=\left(k_{i}^{0}+k_{i}^{z}\right) /\left(p_{N}^{0}+p_{N}^{2}\right)$ is the light-cone momentum fraction of each constituent and $\sum_{i} x_{i}=1, \sum_{i} k_{\perp i}=0$ in the three-quark Fock state.

Note the presence of the asymmetric exponentials that explain how a flavor-spin asymmetry shows up in the $x$-dependence of the quark distributions. The outer quark in the wave-function exponentials (particle 1 or 2 in Eq. (10)) has the product $x \alpha^{2}(1-\mathrm{d})$ in the denominator of the exponential and, with $d>0$, will have a larger average $x$ than the core $\mathrm{u}-\mathrm{d}$ quarks. Since the outer quark has the polarization of the nucleon, one arrives at the following picture of the longitudinal r romentum-space segregation. As a result of the spin-spin interaction in the quark dynamics, there is a u-quark in the proton that dominates the large- $x$ region with the $u-d$ quarks squeezed into the small-x part of the phase space. This has two immediate consequences: the siructure function ratio $F_{2}^{n}(x) / F_{2}^{p}(x)$ drops to $1 / 4$ as $x \rightarrow 1$, and the proton and neutron polarization asymmetry rise to 1 in the large $\mathbf{x}$ limit. Corresponding predictions for the structure functions, both with the QCD evolution (solid) and without (dash-dot), are given in Fig. 3. Now, comparing similarities of the $x$-space and configuration-space distributions, one concludes that, although the $u$-quark in the proton is on average at larger distances from the center of mass than is the d-quark, with its Fourier transform being narrower than that of the d-quark, after the change to light-cone variables, the $u$-quark $x$-distribution is broader than that of the d-quark. Thus, with the use of the light-cone, the apparent disagreement regaiding the sign of the neutron charge radius between the DIS duta at high $Q^{2}$ and the results of elastic electron-nucleon scattering at low $Q^{2}$, widely noted in 
Refs. $[18,19,20]$, can be explained.

I believe that this rather remarkable success of the QCD-inspired quark model suggests that the same spatially dependent spin-spin interaction which breaks the mass degeneracy of the nucleon and delta is also responsible for segregating the quark charge, flavor and spin within the nucleon. Similar conclusions regarding the origin of the structure-function asymmetries have been reached recently by Close and Thomas [21].

This paper owes a great deal to J. Franklin with whorn I engaged in extensive discussions during the initial stage of the work. I also wish to thank R. Carlitz, C. Carlson, E. Henley, N. Mukhopadhyay, K. Nikolaev, and H. Weber for helpful discussions, the Institute for Nuclear Theory at the University of Washington for its hospitality, and the Department of Energy for partial support during the completion of this work. Futhermore, I am grateful to R. Intemann for his remarks on the manuscript and to E. Gawlinski for a help with Temple Graph. 


\section{References}

[1] N. Isgur and G. Karl, Phys. Lett. 72B, 109 (1977); 74B, 353 (1978); Phys. Rev. D18, 4187 (1978); D19, 2653 (1979); D20, 1119 (1979).

[2] C. Hayne and N. Isgur, Phys. Rev. D25, 1944 (1982); S. Capstick and N. Isgur, Phys. Rev. D34, 2809 (1986).

[3] A. Le Yaouanc, L. Oliver, O. Pene, and J.C. Raynal, Phys. Rev. D18, 1591 (1978).

[4] N. Isgur, G. Karl, and R. Koniuk, Phys. Rev. Lett. 41, 1269 (1978).

[5] The "uds" basis was first introduced by J. Franklin, Phys. Rev. 172, 1807 (1968) and recently used in the quark model calculations of Refs. [1] and [2]. In this approach, one notes that by isospin symmetry in the $u-d$ sector it is sufficient to consider the flavor states | uud $>$ and | $d d u>$ for the proton and the neutron, respectively. At the same time one carries out the antisymmetrization (or symmetrization if the color is kept implicit) only with respect to the quantum numbers of the first two identical quarks. Keeping this remark in mind, I will supress altogether the flavor indices of my wave functions.

[6] G. G. Simon, F. Borkowski, Ch. Schmitt, and V. H. Walther, Z. Naturforsch. 35a, (1980).

[7] V. E. Krohn, G. R. Ringo, Phys. Rev. D9, 1305 (1973) and L. Koester et al., Phys. Rev. Lett. 36, 1201 (1976).

[8] R. D. Carlitz, S. D. Ellis and R. Savit, Phys. Lett. 68B, 443 (1977); N. Isgur, Acta Phys. Polon.B8, 1081 (1977). 
[9] M. Ida and R. Kobayashi, Prog. Theor. Phys.36,846 (1966); D. B. Lichtenberg and L. T. Tassie, Fhys. Rev. 155, 1601 (1967); Z. Dziembowski, W. J. Metzger and R. T. Van de Walle, Z. Phys. C10, 231 (1981); S. Fredriksson, M. Jāndel, and T. Larsson, Z. Phys. C14, 35 (1982).

[10] S. J. Brodsky and G. P. Lepage, Phys. Rev. D22, 2157 (1980).

[11] G. Parisi and R. Petrontzio, Phys. Lett 62B, 331 (1976).

[12] R. D. Field, Applications of Perturbative QCD, (Addison-Wesley Publishing Company, New York, 1989) p. 14.7.

[13] R. L. Jaffe and G.G. Ross, Phys. Lett. 93B, 313 (1980).

[14] Z. Dziembowski, Phys. Rev. D37, 768 (1988).

[15] I illustrate the Brodsky-Huang-Lepage prescription with the symmetric Gaussian momentum wave function. In the rest frame $\left(\mathrm{p}_{N}^{+}=p_{N}^{-}=\right.$ $\left.m_{N}, \mathrm{p}_{\perp}=0\right)$ this Schrodinger wave function can be converted to a lightcone one by the following chain of substitutions: $\sum\left(2 m+k^{2} / m\right) \approx$ $2 \sum \sqrt{m^{2}+k^{2}}=\Sigma\left(k^{+}+k^{-}\right)=p_{N}^{+}+\sum\left(m^{2}+k_{\perp}^{2}\right) / x p_{N}^{+}=m_{N}+$ $\sum\left(m^{2}+k_{\perp}^{2}\right) / x m_{N}$. Note that a Gaussian wave function with $<k^{2}>^{1 / 2} \simeq$ $250 \mathrm{MeV}$ and $m \simeq 330 \mathrm{MeV}$ has $\left\langle\left(m^{2}+k^{2}\right)^{1 / 2}>\simeq 414 \mathrm{MeV}\right.$ not far from $<\left(m+k^{2} / 2 m\right)>\simeq 425 \mathrm{MeV}$. For details of the asymmetric case, see Z. Dziembowski and J. Franklin, Phys. Rev. D42, 905 (1990) and references given therein.

[16] A C. Benvenuti et al., Phys. Lett. 237B, 599 (1990).

[17] G. Baum et al., Phys. Rev. Lett. 51, 1135 (1981); J. Ashman et al., Nucl. Phys. B328, 1 (1989). 
[18] D. Parashar and R. S. Kaushal, Phys. Rev. D13, 2684 (1976); A. Niegawa and D. Kiang, Phys. Rev. D14, 3234 (1976).

[19] A. Le Yaouanc, L. Oliver, O. Pene, and J.C. Raynal, Phys. Rev. D18, 1733 (1978).

[20] J. Franklin, Phys. Rev. D21, 241 (1980).

[21] F. E. Close and A. W. Thomas, Phys. Lett. 212B, 227 (1988). 


\section{Figure Captions}

Fig. 1 The neutron charge density.

Fig. 2 The flavor-spin asymmetries.

Fig. 3 DIS structure functions at the initial scale $Q_{0}^{2}$ (dash-dot) and the final scale of $16 \mathrm{GeV}^{2}$ (solid), for $m_{q}=330 \mathrm{MeV}, \alpha=264 \mathrm{MeV}$ and d $=0.372$.

(a) The ratio of the neutron to proton structure functices compared with the data of Ref. [16].

(b) The proton spin asymmetry compared with the data of Ref. [17]. 


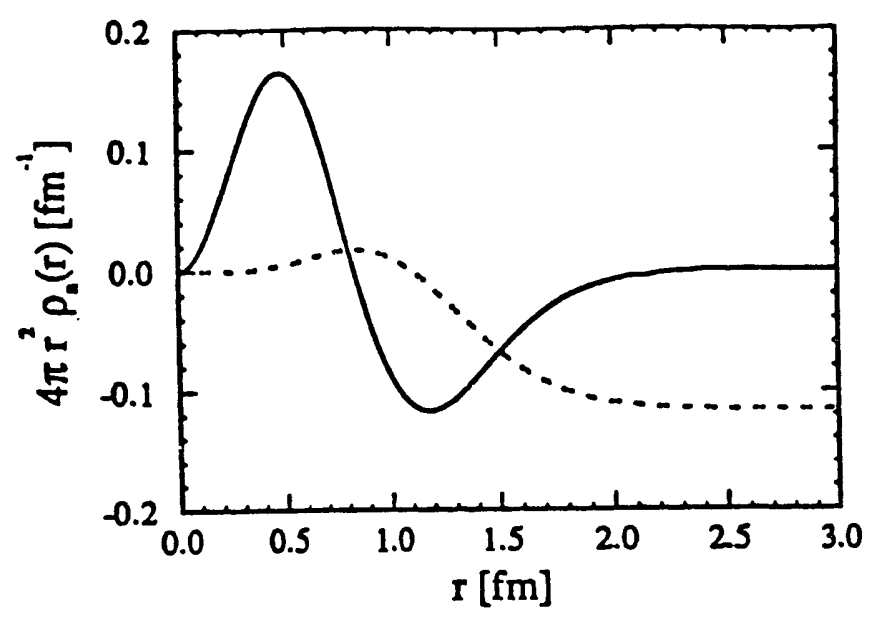

Figure 1

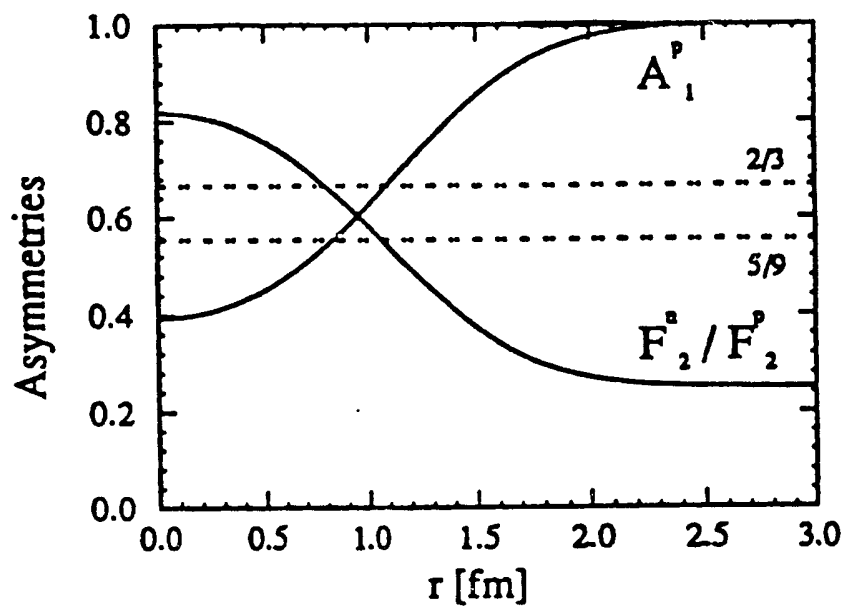

Figure 2
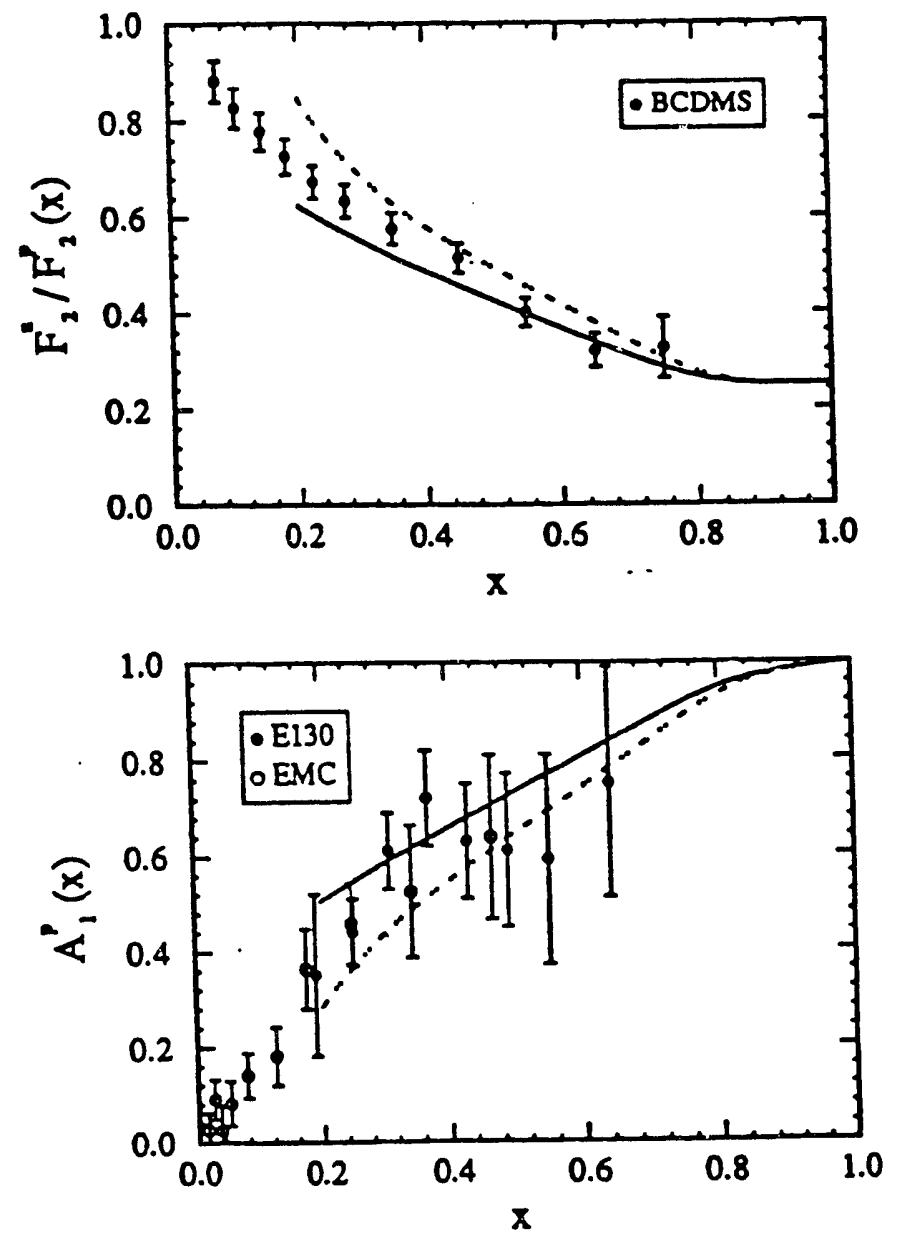

Figure 30 

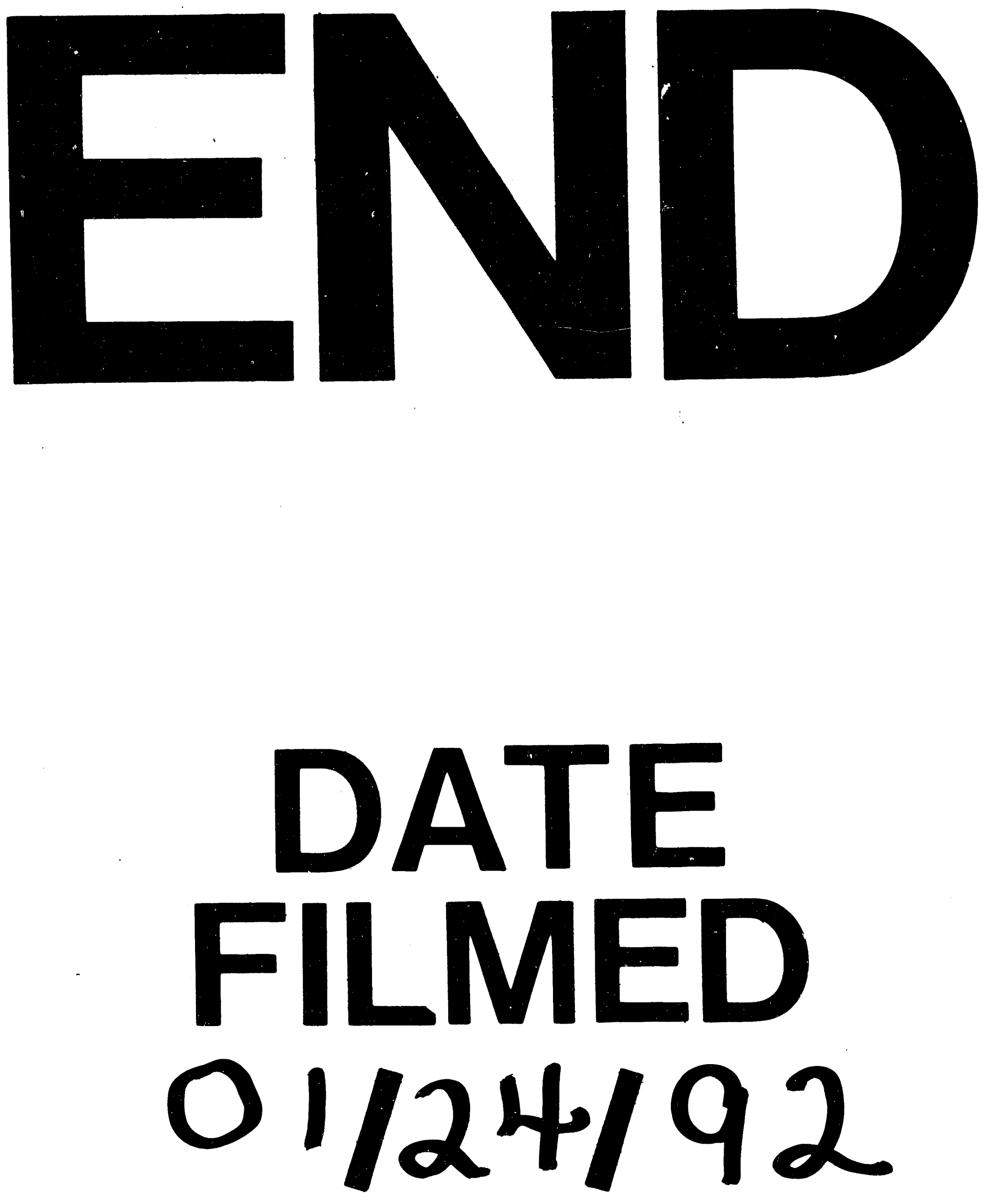

| 
